CAHIERS DE

NARRATOLOGIE

\section{Cahiers de Narratologie}

Analyse et théorie narratives

$27 \mid 2014$

Les bifurcations du récit interactif: continuité ou rupture?

\title{
La narrativité vidéoludique : une question narratologique
}

Marc Marti

\section{CpenEdition}

Journals

Electronic version

URL: http://journals.openedition.org/narratologie/7009

DOI: $10.4000 /$ narratologie.7009

ISSN: 1765-307X

Publisher

LIRCES

Electronic reference

Marc Marti, « La narrativité vidéoludique : une question narratologique », Cahiers de Narratologie [Online], 27 | 2014, Online since 18 December 2014, connection on 19 April 2019. URL : http:// journals.openedition.org/narratologie/7009; DOI : 10.4000/narratologie.7009

This text was automatically generated on 19 April 2019

Article L.111-1 du Code de la propriété intellectuelle. 


\title{
La narrativité vidéoludique : une question narratologique
}

\author{
Marc Marti
}

1 Si l'on part de l'hypothèse que tout jeu vidéo possède le potentiel d'un « récit minimal ", la première question qui se pose est celle de la structure représentée de ce récit ${ }^{1}$. Il n'en demeure pas moins que cette structure visible et réalisée de la narrativité dans le jeu vidéo ne rendrait pas compte de l'intrigue telle que l'envisagent les théories de la réception et de la psychologie cognitive et que, finalement, elle ne viendrait que répéter sur un objet nouveau les limites que l'approche structurale dessinait pour les textes littéraires.

2 Le jeu vidéo fait l'objet d'un intérêt récent de la part de la recherche universitaire, qui y voit un objet nouveau, en particulier pour la narratologie. Il conviendra dans un premier temps de se poser la question du rapport qu'entretient cette science avec ce nouvel objet. Il s'agira de définir la narrativité vidéoludique comme la combinaison entre une intrigue « programmée » et matérielle et l'expérience de cette intrigue par le joueur.

3 A partir de ce constat, nous postulerons l'existence de prototypes vidéoludiques, qui permettraient de décrire des degrés de narrativité variables en fonction des jeux ou des moments de jeu.

4 La distinction de prototypes narratifs nous permettra d'approfondir les rapports entre le jeu et le joueur du point de vue de la narrativité, mais aussi d'établir une typologie où l'articulation entre l'endo-narratif et le narratif occupe une place importante. Elle nous sera utile pour approfondir la notion d'immersion et d'interaction, qui est essentielle dans les jeux vidéo mais aussi en narratologie.

\section{Quelle narratologie pour les jeux vidéo?}

5 La question de narrativité vidéoludique recoupe en apparence les préoccupations des études littéraires sur le récit. Si on admet que la partition qui s'est dessinée au début des games studies entre ce que l'on nommait les ludologues d'une part et les narratologues de 
l'autre est dépassée, la seule façon d'aborder la question est de se servir, sans préjugés, des réflexions issues de ces deux champs de la recherche.

La narratologie a des origines littéraires anciennes et connues. Cependant, cette ancienneté dans le champ scientifique n'a pas abouti à une théorie unique des récits. Sans revenir sur l'ensemble des travaux qui s'y rattachent, ce qui demanderait une synthèse qui dépasse le cadre de cette contribution, la narratologie des débuts a privilégié l'analyse des phénomènes textuels, qu'il s'agisse des techniques de narration ou des lois qui régissent l'univers raconté 2 . La distinction des deux champs était théorique, puisque l'un était difficilement envisageable sans la prise en compte de l'autre. Le terme "récit» tendait ainsi à désigner l'ensemble constitué par l'histoire racontée (histoire) et la façon dont elle était racontée (narration).

7 Cependant, la limitation de ces approches a fait revenir sur le devant de la scène la problématique $\mathrm{du}$ sens des récits, qui supposait un déplacement de la question narratologique. Si la narratologie thématique et la narratologie modale répondaient principalement à la question «comment fonctionne un récit?», la problématique du « pourquoi le récit?» restait en grande partie négligée. Cette question était en fait double et pouvait se formuler en deux interrogations complémentaires : " pourquoi écrit-on des récits? » et " pourquoi lit-on des récits? ». Les travaux récents de Raphaël Baroni et leur aboutissement au concept de tension narrative essaient de répondre principalement à la seconde question ${ }^{3}$. La première question, celle de la motivation de la production de récit, reste plus marginale dans le cadre des études littéraires. Elle entre plutôt dans le cadre de la littérature et de ses rapports avec le contexte historique, ainsi que de possibles applications dans les sciences sociales ou la génétique textuelle.

Ces perspectives offertes par la narratologie littéraire nous semblent intéressantes au moment d'aborder l'analyse de l'intrigue dans le jeu vidéo. Posées en ces termes, elles permettent dès le départ de prendre en compte non seulement la création et le fonctionnement de la structure matérielle du jeu, mais aussi l'expérience narrative qu'il suppose, indissociable de l'expérience du jeu. Cependant, comme le rappelle Julien Rueff, la position des ludologues rappelle la complexité de ce media; ceux-ci « reconnaissent l'existence possible d'éléments narratifs dans les jeux vidéo [mais] ils contestent la légitimité de la narratologie, en soulignant les insuffisances d'une approche exclusivement centrée sur les aspects narratifs, aveugle aux spécificités de ces loisirs numériques ${ }^{4}$ ». Si cette position, résumée de façon tranchée, reste valable, on peut cependant émettre l'hypothèse que chaque jeu possède une proportion plus ou moins grande de narrativité. Cette narrativité découlera de la combinaison d'une intrigue matérielle, programmée, avec l'expérience que le joueur aura de cette intrigue.

Cependant, cette hypothèse de départ doit être nuancée. En effet cette narrativité pourrait avoir une nature modulable, et dépendre en particulier de la relation qui s'établit entre la thématique du jeu, sa structure matérielle, la façon de jouer qu'elle impose et par conséquent l'expérience qu'en retire le joueur. Pour cette raison, nous allons postuler l'existence de prototypes vidéoludiques, qui permettraient de décrire des degrés de narrativité différents en fonction des jeux ou des moments de jeux. Le postulat sera que tout jeu s'appuie sur un prototype narratif dominant, qui n'est pas forcément exclusif des autres. 


\section{Narrativité vidéoludique : du genre au prototype}

10 Avant d'aborder la question des prototypes, on peut convenir que la notion de genre est assez centrale dans la catégorisation des jeux vidéo. Les produits de l'industrie vidéoludique sont constamment définis en termes de genre ${ }^{5}$.

Loin de se cantonner à des classifications savantes et oiseuses, [le genre] intervient en profondeur dans le processus de création, mais aussi dans le processus de réception et de consommation de l'œuvre.

11 Dans ce cadre, le genre est presque toujours une catégorie qui s'élabore $a$ priori et non $a$ posteriori. En effet, le choix et/ou la programmation d'un moteur de jeu spécifique, le graphisme qui donnera la thématique orientent fortement la réception de l'œuvre. Par ailleurs, les stratégies marketing fondées sur le ciblage font du genre a priori un moyen d'optimiser les ventes. Le genre est donc d'abord une classification empirique opératoire si l'on se place du point de vue de la production.

12 Du côté des universitaires, Dominic Arsenault, qui a travaillé sur les formes et les mutations du genre dans le jeu vidéo, a cependant montré que la création d'une typologie des genres vidéoludiques sous la forme "d'un tableau bien droit, bien organisé qui regrouperait tous les critères employés pour affilier tous les jeux à tous leurs genres correctement » est une entreprise vaine ${ }^{6}$. La notion même de genre résiste à ce type de projet. Le jeu vidéo se caractérise précisément par une multiplicité de critères très évolutifs et ne peut être réduit à une typologie rigide ${ }^{7}$ :

C'est en considérant le genre comme phénomène discursif plutôt que structurel, et comme cristallisation temporaire d'un consensus culturel commun, qu'on peut saisir sa nature propre comme phénomène discursif et fondamentalement flou. [...].

13 L'histoire d'un genre vidéoludique repose sur les rapports entre son émergence les imitations auxquelles il donne lieu, ses possibles réitérations (les suites) et les innovations techniques.

14 L'histoire de ces genres a été peu explorée, si on excepte la thèse de Jonathan Lessard qui s'est intéressé à l'histoire formelle du jeu d'aventure sur ordinateur entre 1976 et $1999^{\circ}$. Ce travail pionnier retrace de façon convaincante la naissance, le développement et la fin d'un genre. Son analyse prend en compte des critères aussi disparates que l'évolution des interfaces, le changement des équipements informatiques, l'arrivée d'un public de néophytes ou le poids de la presse spécialisée. Dans les années 2000, le modèle narratif du jeu d'aventure fusionne en quelque sorte avec le jeu d'action, qui va définitivement l'assimiler 9 . Pour Lessard, le genre pourrait reposer en partie sur une expérience narrative homogène, malgré les innovations matérielles. Pour lui, il y aurait donc une architecture narrative qui serait la constante structurelle générique et historique.

Cette conclusion rejoint les remarques que nous avions faites dans un travail précédent ${ }^{10}$. Cela nous avait amené à considérer les jeux par prototypes narratifs, à partir d'une approche à la fois structurale et pragmatique. La typologie théorique obtenue allait du jeu " à récit complet » au jeu « à contenu narratif faible », en passant par les " jeux temporels et évolutifs ». Cette approche posait la question de la structure scénaristique fondatrice de chaque type de jeu en tenant compte de deux éléments indispensables dans l'analyse vidéoludique qui sont «l'objectif» du jeu (l'arrivée au dénouement en termes narratologique) et les moyens mis en œuvre (jouables) pour l'atteindre. Ces deux préalables posés, on pouvait envisager non pas trois genres mais trois prototypes 
narratifs. L'intérêt de cette typologie est qu'elle permet de s'interroger sur le rapport entre l'intrigue structurelle et la façon dont le joueur est amené à l'aborder, à la « jouer ».

\section{Trois prototypes, trois narrativités ?}

Si on envisage les jeux à partir de trois prototypes narratifs, cela signifie que l'on postule que la narrativité est une variable dans le jeu vidéo. Sa variabilité va dépendre d'abord du jeu, pris comme une unité, de la même façon que l'on parle d'un texte en narratologie. Cependant, cette variabilité sera aussi soumise à la façon dont on jouera le jeu. En effet, certains jeux proposent des moments plus ou moins chargés de narrativité, tout comme ils peuvent proposer aussi différentes façons de jouer, qui correspondent à des finalités diverses pour le joueur.

Par ailleurs, postuler l'existence de trois prototypes narratifs reviendra, comme nous allons le voir, à établir des degrés de narrativité, qui peuvent se fonder sur les relations entre narratif et endo-narratif.

\section{Narratif ou endo-narratif : les jeux à contenu narratif faible}

De l'aveu même des concepteurs, un certain nombre de jeux sont créés sans scénaristes, ce qui révèle parfaitement l'aspect secondaire que joue la trame narrative dans ces réalisations ${ }^{11}$. D'une certaine façon, ils n'ont pas besoin de raconter très précisément une histoire pour donner envie de jouer. La série des jeux de plateforme Super Mario (Nintendo) repose par exemple sur une intrigue des plus simplistes qui ne sert pas réellement à jouer. Ce minimalisme explique en partie les qualifications péjoratives qui accablent cet univers, ce qui a conduit par ailleurs un certain nombre de créateurs à remettre en question la place du récit dans le jeu vidéo ${ }^{12}$.

Cependant, cette façon de voir les choses est fortement biaisée par quelques préjugés que véhiculent sans le savoir les narratologues, qui ont forgé leurs outils à partir des œuvres littéraires. Il ne viendrait à l'idée de personne de reprocher au roman la pauvreté de ses graphismes ou la faiblesse de son interactivité. C'est la même chose pour le jeu vidéo, qui ne peut être exclusivement jugé sur le critère de son intrigue ou de la richesse de son univers diégétique. Réduite à un récit simpliste et minimaliste, l'intrigue n'est la plupart du temps qu'un prétexte visant à donner un vague cadre narratif et un but à une succession d'actions réalisées par le joueur. En fait, ce prototype, du point de vue de la narratologie, mobiliserait presque uniquement les compétences endo-narratives du joueur. Selon Gervais ${ }^{13}$ :

[...] l'endo-narratif, défini comme en-deçà narratif, est cette frange théorique étroite qui permet de rendre compte des processus d'identification des actions représentées, avant leur intégration à une narration.

Dans les jeux de plateforme ou de combat, le joueur activerait principalement des compétences endo-narratives pour réaliser des actions qui relèvent "d'un ordre générique stéréotypé ", sans nécessairement les considérer dans une intrigue. Cette remarque rejoindrait la conclusion Raphaël Baroni sur les rapports entre l'endo-narratif et le narratif ${ }^{14}$. Concrètement, le joueur essaie de réaliser le mieux possible des actions comme sauter, courir, attraper, tirer, frapper, accélérer, freiner, sans forcément se préoccuper de leur intégration à une narration. 
En effet, ces jeux « à contenu narratif faible » ou endo-narratifs, reposent essentiellement sur l'identification d'un objectif de joueur chiffrable et assimilable à des performances physiques (réflexe, motricité, vitesse). Les jeux de combat, de course, de réflexion, de sport, de plateforme ainsi que les jeux de cible constituent de bons exemples de ce premier prototype. Le contenu narratif est faible, car c'est avant tout le score atteint qui constitue l'objectif du joueur. Très souvent, ils proposent des écrans où le score, le temps, ou des éléments du même type occupent une place centrale pendant la partie.

\section{Copie d'écran, Mariokart Game Cube $2006^{15}$}

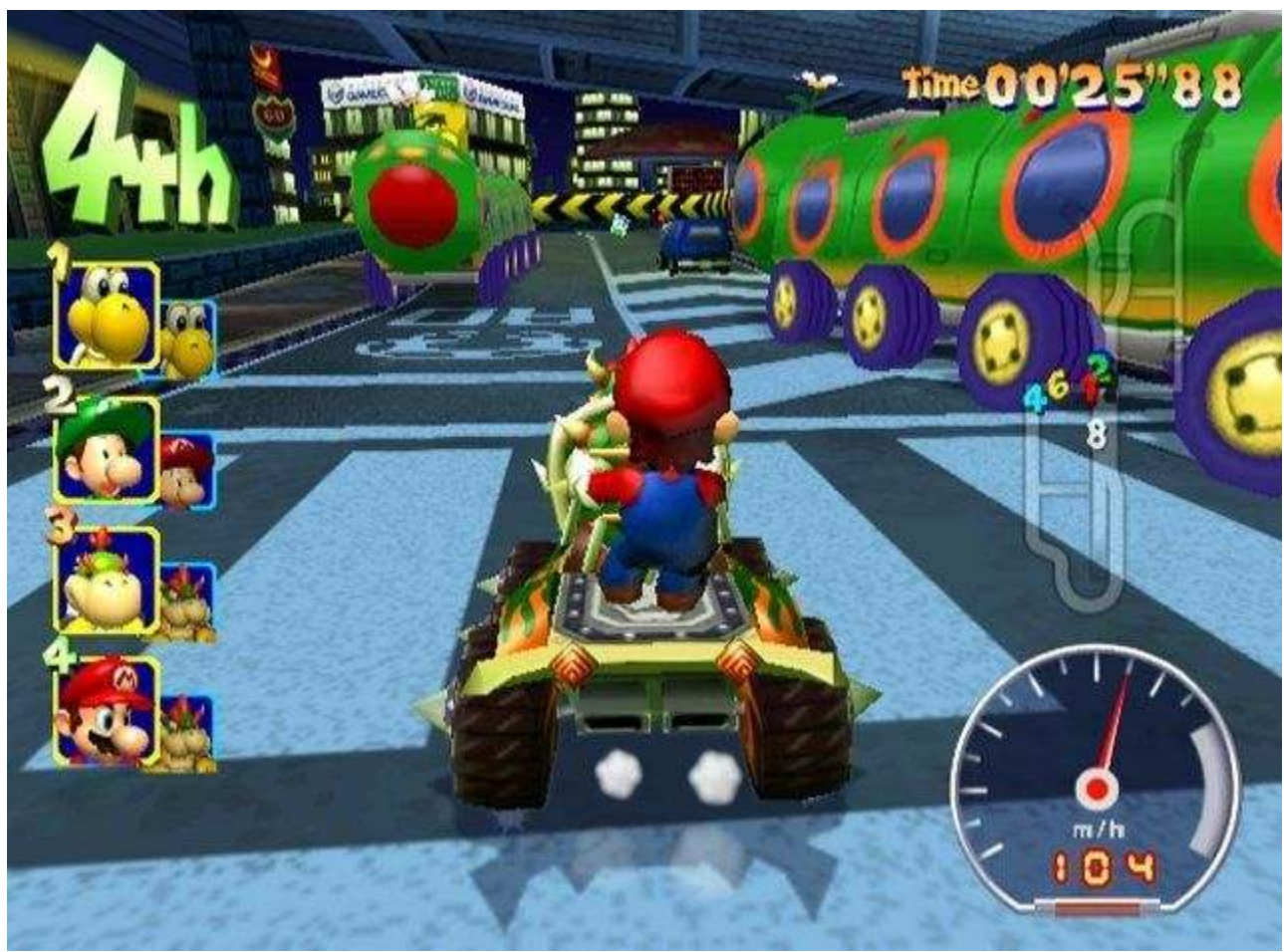

Globalement, la faible narrativité se concerne autant la structure de l'histoire que la jouabilité. Le joueur n'y réalise pas une intrigue, qui d'ailleurs parfois n'existe pas. Il s'exerce plutôt au plaisir de la performance physique, se rapprochant bien plus du sport que de la lecture. Cette reconnaissance sportive est d'ailleurs en marche dans plusieurs pays, dont les Etats-Unis ${ }^{16}$. Les notions de tournois, combats, compétitions, sont d'ailleurs très prégnantes et dominent la pratique socialisée de ces jeux. Par ailleurs, on remarque que l'immersion physique y est de plus en plus poussée, avec des dispositifs visant à rendre sensibles les mouvements du corps au-delà de la main.

\section{Jeux « à récit complet » : du récit fermé au récit ouvert}

\section{Des caractéristiques communes}

Les jeux «à récit complet » semblent les plus proches des prototypes narratifs définis par la littérature. Une partie d'entre eux a donné lieu, pour les plus célèbres, à des adaptations cinématographiques qui confirment, d'une certaine façon, l'existence d'une narrativité extrapolable ${ }^{17}$. Les FPS First Person Shooter ou jeu de tir à la première personne, l'aventure-action ou les récits interactifs dans toutes leurs déclinaisons thématiques en sont les meilleurs exemples. 
Dans ces jeux à récit complet, scénarisés préalablement, l'intrigue prend un autre sens, plus intéressant du point de vue du narratologue. Le joueur garde l'impression qu'il est dans une intrigue "ficelée" dans le sens où il y a une continuité spatiale de sa progression, suggérée par des graphismes qui ancrent le récit dans une topographie et une ambiance spécifique, dans lesquelles il va évoluer.

Les compétences narratives mobilisées sont plus fortes, car l'idée de progression vers un but/dénouement est très marquée. Malgré ces caractéristiques communes, le prototype peut fonctionner suivant deux variantes : le récit fermé et le récit ouvert.

\section{Le récit fermé}

Les FPS ou les PMT (Porte-Monstre-Trésor) sont de bons exemples de récit fermé. Dans ces jeux, les résultats d'un épisode constituent la base du suivant, mais surtout, chaque épisode n'a qu'un seul dénouement possible. Le climax est atteint au moment du final, très souvent par un combat contre un adversaire supérieur aux autres. Dans beaucoup de jeux scénarisés, les actions précédentes «s'accumulent » non pas uniquement sous forme de score, mais sous la forme d'objets (armes, armures, trousses de secours, etc...) ou d'expérience qui permettront d'affronter correctement les niveaux supérieurs. Le classique Doom II (ID, 1994) demandait déjà d'arriver au niveau supérieur le mieux armé possible et "en bon état » afin de pouvoir affronter des adversaires de plus en plus nombreux, de plus en plus résistants et dotés d'un pouvoir de destruction de plus en plus létal. La temporalité, qui se traduit scénaristiquement par l'acquisition de compétences supérieures, joue donc un rôle déterminant.

On remarque cependant que ce sont souvent des éléments non-jouables qui viennent encadrer l'action pure. Ceux-ci introduisent la situation initiale et le nouement que l'on retrouvera dans des cinématiques, sur les jaquettes, voire dans les manuels d'instructions. Dans les jeux plus contemporains, distribués sur DVD, l'ajout d'une ou plusieurs cinématiques d'entrée et de sortie renforce cette impression d'intrigue complète, en permettant de visionner la situation initiale et finale. Certains jeux - par exemple Diablo III (Blizzard, 2012) - proposent des cinématiques intermédiaires, à la fin de chaque niveau (voir http://www.youtube.com/watch?v=0jNcUZgGYdo).

Ces cinématiques ne sont pas anodines et font l'objet de toutes les attentions, car leur objectif principal est d'ancrer les actions jouées dans un cadre narratif prégnant et donc immersif. Il s'agit avant tout d'intégrer les épisodes joués, qui sont principalement du ressort de l'endo-narratif, dans une progression narrative explicite. La cinématique rapproche ainsi le jeu du cinéma, genre narratif par excellence. Il n'est pas non plus anodin qu'une partie des graphistes des jeux vidéo travaillent aussi pour le cinéma et la BD. Par exemple le dessinateur Gérald Brom a travaillé à la fois pour ID Software (Doom II, Heretic II) et le cinéma (Van Helsing, Ghost of Mars). La tendance dans les derniers blockbusters vidéoludiques est aussi d'utiliser des acteurs connus pour "tourner" les animations ${ }^{18}$. Ces grosses productions imitent aussi le cinéma en publiant des bandesannonces, qui insistent essentiellement sur le scénario du jeu et non sur ses aspects « techniques ", comme le moteur ou les actions possibles pour le joueur, bien que ceux-ci puissent être évoqués. Le trailer de Wolfenstein New Order (Machinegames-Bethesda, 2014), dont la sortie était prévue en février 2014, a été diffusé dès juin 2013 (voir http:// www.youtube.com/watch?v=WfeIBXtiINo). 

éléments de suspense ou de curiosité qu'elle pourrait mettre en œuvre. Les joueurs semblent d'ailleurs tenir beaucoup à une intrigue et à un monde pour s'investir dans un jeu. Quand le studio 11 Bits a annoncé par provocation qu'il proposait en prévente un jeu sans en révéler le pitch (terme utilisé fréquemment pour désigner le résumé de l'intrigue), les réactions sur Internet ont été globalement très négatives, alors que l'annonce était accompagnée d'un teaser visant à intriguer le public, mais sans qu'il soit dit que le teaser en question correspondrait à l'univers du futur jeu ${ }^{23}$. Le teaser constitue pourtant une accroche, mais pas un pitch complet (http://www.youtube.com/watch?v=SVbaqQIySE8) sérielle. Le joueur connaît à l'avance le dénouement, qui est celui de la victoire de son avatar puisque le jeu a été écrit ainsi. Le plaisir va se trouver principalement dans la façon dont le jeu va lui permettre de s'immerger et la façon dont il va arriver au terme du jeu. Plus exactement la façon dont on va le conduire et dont il va atteindre lui-même ce point final. Selon les catégories définies par Raphaël Baroni pour les textes narratifs, c'est le «suspense moyen » qui domine : le joueur connaît le dénouement et détourne "son intérêt de l'issue vers les circonstances qui mèneront au dénouement attendu ${ }^{24}$ ». Le joueur qui connaît la fin (parce que c'est la loi du genre, comme dans les FPS), dans le sens où il sait qu'il va "gagner " plus ou moins rapidement en fonction de la durée de vie du jeu, se retrouve dans la position du lecteur de romans de genre. Ce ne sera pas tant la fin qui comptera, mais les moyens mis en œuvre pour y parvenir.

Bien que plus élaborées que les intrigues des jeux de plateforme, qui privilégient l'immersion physique, les intrigues des FPS ou de PMT n'en demeurent pas moins classiques dans leur dimension structurale. Il est évident qu'au cours des dernières années, l'évolution matérielle, l'augmentation croissante des budgets et l'apprentissage culturel des joueurs ont fait évoluer vers le haut les attentes en terme d'intrigue. De plus 
en plus celle-ci doit être immersive ou " crédible ", dans le sens où le joueur doit se sentir impliqué narrativement (émotionnellement) dans l'intrigue dont il va jouer les épisodes. On pourrait considérer que l'intrigue n'est plus actuellement secondaire par rapport à la jouabilité (d'ordre matériel). Un «bon » FPS ou PMT ne repose pas nécessairement sur une intrigue complexe mais celle-ci doit être soignée. Elle a pour fonction essentielle de justifier et de compléter les mécanismes d'immersion que sont les coordonnées spatiotemporelles et la finalité narrative des actions. Elle servira à inspirer les graphistes, qui s'orienteront, en fonction des besoins, vers la SF, les univers glauques, les décors historiques, etc. Mais c'est la qualité de ces décors qui contribuera aussi à la qualité de l'immersion.

Un cas exemplaire est celui du FPS Call of Duty dont la série a débutée en 2003. Le premier opus mettait en scène une intrigue se déroulant au cours de la seconde guerre mondiale. Cependant, cette thématique s'est usée avec le temps, d'autant que la franchise concurrente, Medal of Honor avait décliné à peu près tous les théâtres d'opérations du conflit. En conservant donc des principes structuraux quasi identiques, la thématique a évolué vers des conflits contemporains et dans certains modes, les adversaires ne sont plus des soldats mais des zombies, issus d'expérimentations nazies. L'intrigue ainsi déplacée dans le temps donne une pseudo-nouveauté au jeu qui continue à reposer sur des principes scénaristiques éprouvés et qui se sert parfois de sa propre histoire, les nouveaux opus reposant sur les récits des premiers, contribuant ainsi à construire un effet-monde. Le déplacement temporel et spatial de la diégèse vient remplir la fonction que nous avons analysée, en justifiant de nouveaux mondes graphiques sur lesquels reposent réellement la nouveauté.

Il faut aussi reconnaître que dans certains cas, la nouveauté ne va pas au-delà du simple remake graphique. Duke Nukem, devenu un classique depuis sa première version FPS en 1996 n'a pratiquement pas changé de scénario pour la version Duke Nukem forever sortie en 2011. La principale innovation a consisté à passer à un univers graphique plus contemporain. La bande annonce de ce classique regorge de stéréotypes et ne fait que répéter (scénaristiquement parlant) le récit des versions précédentes (voir http:// www.youtube.com/watch?v=wVuuyRGB_BA) .

$\mathrm{Au}$ bout du compte, le jeu à récit complet, fermé et donc unique, se rapproche des formes narratives classiques. Cependant, pour «bien jouer», le joueur doit retrouver par tâtonnements (qui se soldent par des game over), le meilleur moyen de faire coïncider l'intrigue qu'il joue avec l'intrigue prévue par les concepteurs du jeu. Le joueur devra réaliser les actions exactes prévues par les concepteurs du jeu afin de parvenir au dénouement attendu. C'est justement sur ce terrain que la différence se marque avec les jeux ouverts, qui proposent un récit complet mais qui sera mené d'une façon différente.

\section{Les jeux à récit ouvert}

37 La narrativité des récits ouverts est directement issue de la mise en application de la théorie des possibles narratifs: «le récit se construirait suivant une série d'embranchements binaires consacrant le déroulement ou le non-déroulement d'une possibilité, d'une virtualitée ${ }^{25} »$.

38 Au lieu d'imposer au joueur une intrigue unique, ces jeux laissent la possibilité de choix multiples et offrent ainsi la possibilité de mener à bien des intrigues complexes. Cette complexité est à comprendre comme une ouverture des possibles narratifs: le choix 
opéré par le joueur aux « embranchements » ouvre des possibilités narratives différentes. Là où l'intrigue classique repose chaque fois sur un seul possible narratif réalisable, le récit ouvert laisse la possibilité de bifurcation, d'actualisation de récits qui auraient eu le statut de « récits fantômes » dans un scénario classique ${ }^{26}$. Ainsi les possibles narratifs non explorés (car non explorables dans un scénario classique) ne sont plus des spéculations mais des récits jouables.

Heavy rain (2010), développé par la société française Quantic Dream, illustre parfaitement cette forme de narrativité. Le joueur peut incarner quatre avatars différents. L'intrigue repose sur la recherche d'un tueur en série qui a enlevé un enfant. Le jeu, qui a connu un succès important, propose dix-huit fins alternatives en fonction des choix que le joueur fait en amont du dénouement. Considéré comme un récit interactif, Heavy rain propose aussi des combats et des courses poursuites.

Il faut cependant noter que ce type de jeu repose toujours sur un nouement fixe et unique. Les possibilités multiples s'appliquent à l'action et $\mathrm{au}(\mathrm{x})$ dénouement(s) qui en découle(nt). Bien que très présente dans les jeux dits d'aventures ou les récits interactifs, cette ouverture des possibles narratifs, qui apporte une plus-value narrative indéniable, est aussi utilisée dans les dernières versions des FPS. Call of Duty: Black Ops II en mode campagne offre ainsi huit dénouements alternatifs. Cependant, il ne s'agit que de huit cinématiques différentes, qui sont conditionnées à la mort ou à la survie d'une partie des personnages croisés par le joueur pendant les épisodes qui le conduisent au dénouement. La progression de l'action reste très linéaire et n'est pas soumise à des choix, comme dans le récit interactif.

41 La narrativité ouverte sert d'argument commercial pour mettre en avant la durabilité du jeu. Le joueur sera tenté d'explorer les alternatives et le jeu aura une durée plus importante que dans le cas d'un récit fermé. On remarquera que c'est la variété du dénouement et des moyens d'y parvenir qui apportent de la nouveauté pour le joueur. Les graphismes jouent bien entendu un rôle important dans l'immersion. Cependant, celle-ci dépend encore plus fortement de l'implication du joueur dans la trame narrative plutôt que dans l'action. Ces intrigues ouvertes fonctionnent toujours sur le principe d'un dénouement en climax. Cette notion de climax sera pratiquement absente par contre des jeux évolutifs.

\section{Les jeux évolutifs}

Ces jeux s'éloignent des modèles canoniques du récit, en particulier si l'on examine la position qu'y occupe le joueur. Celui-ci n'est plus «guidé » par une trame narrative (unique ou multiple) d'enchaînement des niveaux ou des épreuves jusqu'à un climax final qui constituerait la «fin» théorique du jeu. Il est au contraire en grande partie laissé libre grâce aux possibilités nombreuses d'interactions qui lui sont offertes.

Ces interactions peuvent être "naturelles", comme dans le cas des jeux en ligne multijoueurs (MMORPG, Jeux de rôle en ligne massivement multi-joueurs) ou bien « artificielles », créées par une IA (intelligence artificielle). Le jeu n'a généralement plus de fin. Les développeurs ont pris en compte les énormes possibilités narratives qu'offrait ce schéma pour des jeux conçus au départ pour un seul joueur. Le très polémique GTA (Grand Theft Auto) illustre cette possibilité. Dans ce jeu, les missions variées doivent être complétées pour progresser dans le scénario et pouvoir jouer d'autres missions. Ces missions apportent des compensations, comme le déblocage de zones de la ville, des 
nouvelles armes ou de l'argent. Ces apports permettent de multiples possibilités, comme acheter des propriétés ou investir en bourse, autant d'actions qui peuvent avoir une incidence dans la suite du jeu. Le joueur intervient dans l'histoire comme il le souhaite car le scénario n'est pas linéaire. Il est aussi possible de vagabonder dans l'univers du jeu sans se soucier de l'histoire, et d'interagir avec les « éléments » que l'on croise : obtenir des véhicules, souvent illégalement, voler de l'argent à un passant, etc.

Le recours à l'illégalité a aussi des conséquences sur la façon dont se développera l'environnement. Plus le joueur aura commis d'actes illégaux, plus il sera traqué par la police. Cet exemple montre que c'est l'accumulation des interactions avec l'environnement (donc l'expérience temporelle) qui modifie l'environnement et les possibilités narratives qui en découlent. Les MMPORG répondent aussi à ce principe narratif avec quelques particularités. L'interaction se fait principalement entre les joueurs connectés et non entre le joueur et l'IA. Par ailleurs, celui-ci évolue dans un monde persistant, c'est-à-dire un monde qui continue à changer hors connexion, sous l'action des autres joueurs connectés. Dans ce modèle, c'est principalement l'accumulation d'une expérience temporelle et narrative qui va ouvrir de nouvelles possibilités pour le joueur. L'objectif n'est plus d'arriver à un dénouement unique, mais de faire progresser son avatar. La jouabilité repose bien plus sur l'avatar et l'augmentation de ses compétences que sur l'intrigue. Théoriquement ces jeux « n'ont pas de fin ». Au regard de la distinction que nous avons opérée entre compétences endonarratives et narratives, leur finalité est d'enchaîner des épisodes, qui aboutissent à des dénouements. A partir de ces dénouements, d'autres épisodes voient le jour, mais l'ensemble ne poursuit aucun dénouement ultime.

Comme l'indique Régis Jaulin, «dans le cas des jeux de rôles en ligne, ils reprennent souvent une histoire préexistante, encadrée par une licence : le futur Matrix, Le Seigneur des anneaux en sont deux exemples. En tout cas, il s'agit rarement de créer de but en blanc un scénario linéaire, mais plutôt de constituer un univers cohérent dans lequel va baigner le jeu ${ }^{27} »$.

\section{Le joueur, clé de l'intrigue vidéoludique?}

L'analyse que nous avons menée jusqu'à présent a surtout consisté à mesurer la narrativité des jeux vidéo à l'aune de conceptions issues des médias linéaires. Pour pertinente qu'elle soit, cette analyse nous semble incomplète, en particulier parce qu'elle ne rend pas totalement compte d'un phénomène que souligne Cécile Pearce en indiquant que «le joueur se retrouve dans une expérience ludique qui lui permet d'acquérir des techniques de création d'une dynamique dramatique ${ }^{28} »$.

Cette remarque supposerait que l'on admette que l'intrigue vidéoludique "jouée », ou "vue de l'intérieur» serait narrativement plus immersive que l'intrigue linéaire du cinéma ou de la littérature. En effet, dans ce cas, le joueur choisit ce que va faire l'avatar et explore les impossibles narratifs. Leur reconnaissance (comme on reconnaît un lieu) donnerait au joueur des compétences dans la dynamique narrative propre au jeu. Cependant, il nous semble ici que si l'on ne parle que de compétences, la différence avec les formes narratives classiques est très faible et ne repose que sur un changement de média. Par contre, si, comme Cécile Pearce, on parle de " techniques », il devient évident que les jeux, au regard de leur principe même de fonctionnement ou de leur environnement technique, font endosser au joueur le rôle d'auteur. 


\section{Le joueur, auteur de récit dans le jeu} joueur est incité, non pas à s'immerger dans une intrigue produite par un autre, mais à s'impliquer dans la création d'une intrigue qu'il va lui même conduire, tout en se conformant aux limites techniques de l'environnement. Ce sont des jeux qui, à des degrés divers, placent le joueur dans un rôle d'auteur à l'intérieur du jeu : la finalité même du jeu est de produire une histoire et non de jouer une histoire. Cette pratique peut être en partie rapprochée de celles qui vont au-delà, en plaçant complètement le joueur dans un rôle d'auteur autonome.

\section{Le joueur auteur de jeu et de récits de jeu}

$51 \quad$ Les jeux évolutifs tendent à placer le joueur dans la position d'auteur d'intrigues. Deux autres pratiques péri-narratives se sont par ailleurs développées, et font des joueurs soit des auteurs de jeux, soit des auteurs de récits de jeu.

De nombreux FPS, dont la naissance a pratiquement coïncidé avec le début du développement de l'Internet, offrent la possibilité pour les joueurs de construire leurs propres niveaux de jeu, à partir d'un éditeur de niveau. Le niveau (ou map) correspond en général dans le FPS à un épisode de l'intrigue générale. L'apparition de ces niveaux fabriqués par les joueurs désarticule l'intrigue et l'éclate en autant d'épisodes potentiels endo-narratifs, qui se raccrochent à l'intrigue principale uniquement par une similitude graphique et actionnelle, ainsi que par la connaissance de "l'histoire de départ». Ces prolongements jouables du jeu central, qui font du joueur un « auteur » rappellent dans une certaine mesure les fanfictions propres aux franchises transmédiatiques ${ }^{31}$. 

scriptée et une narrativité dynamique produite par l'interaction du joueur avec le jeu. La combinaison des deux constitue la narrativité vidéoludique, un concept qui reste encore à affiner et qui pourrait même s'exprimer au pluriel.

Nous avons postulé, en tant que narratologue, que le jeu vidéo reposait toujours, mais à des degrés très divers, sur la narrativité. Selon nous, c'est d'abord la part entre compétences narratives et endo-narratives qui permet de déterminer cette narrativité. Le joueur active les unes et les autres en fonction des moments du jeu et du genre auquel il appartient.

On pourrait provisoirement conclure en affirmant que le joueur «joue » avant tout en mobilisant prioritairement des savoirs pré-requis ou capitalisables comme les compétences endo-narratives, qui lui permettent d'avancer dans un jeu. Pour autant l'expérience ludique ne repose jamais exclusivement sur ces savoirs. Elle possède toujours une dimension narrative variable en fonction de genres vidéoludiques et des moments de la partie. 


\begin{tabular}{|c|c|c|c|c|c|c|}
\hline \multirow{4}{*}{\begin{tabular}{|l|} 
fort \\
moyen \\
faible \\
\end{tabular}} & & & & & & \\
\hline & & & \multicolumn{4}{|c|}{ Prototypes } \\
\hline & & & endo-narratif & \multicolumn{2}{|c|}{ récit complet } & évolutif \\
\hline & & & & fermé & ouvert & \\
\hline \multirow{9}{*}{ Narrativité } & \multirow{3}{*}{ scénarisation } & schéma canonique complet & & & & \\
\hline & & possibles narratifs & & & & \\
\hline & & effet de fiction & & & & \\
\hline & \multirow{3}{*}{ graphismes } & résolution & & & & \\
\hline & & effet monde & & & & \\
\hline & & effet cinéma (mimétisme, acteur) & & & & \\
\hline & \multirow{3}{*}{ action du joueur } & implication dans l'action & & & & \\
\hline & & implication dans le scénario & & & & \\
\hline & & \begin{tabular}{|l|} 
générateur d'intrigue \\
\end{tabular} & & & & \\
\hline
\end{tabular}

\section{NOTES}

1. Pour le concept de récit minimal, voir Bedrane Sabrinelle, Revaz Françoise, Viegnes Michel (éds), Le récit minimal, Paris, Presses de la Sorbonne Nouvelle, 2012.

2. Nous renvoyons à l'étude classique de Claude Brémond, "La logique des possibles narratifs ", Communication, ${ }^{\circ} 8$, Paris, Seuil, 1981, pp. 66-83.

3. Baroni, Raphaël, 2007, La tension narrative. Suspense, curiosité et surprise, Paris, Seuil.

4. Rueff, Julien, « Où sont les "games studies" ", Réseaux, 2008, pp 141-146, voir en particulier les pages 148-149.

5. Letourneux, Matthieu, p. 39, in «La question du genre dans les jeux vidéo », Le game design de jeux vidéo : approches de l'expression vidéoludique, Paris, L'Harmattan, 2006, pp. 39-54.

6. Dominic Arsenault, Des typologies mécaniques à l'expérience esthétique Fonctions et mutations du genre dans le jeu vidéo, Montreal, 2011, p. 15. Thèse en ligne, consultée le 15/11/2013, URL: https://papyrus.bib.umontreal.ca/xmlui/bitstream/handle/1866/5873/

Arsenault_Dominic_2011_these.pdf?sequence $=2$

7. Dominic Arsenault, Op. cit., pp. 333-334.

8. Jonathan Lessard, Histoire formelle du jeu d'aventure sur ordinateur (le cas de l'Amérique du Nord de 1976-1999), Montréal, 2013.

9. Jonathan Lessard, Op. cit., p. 246.

10. Marti, Marc, "Jeux vidéo et logiques narratives ", Espaces et temps des jeux vidéos, éditions Questions théoriques, Paris, 2012, pp. 73-91.

11. Régis Jaulin et al., "Les ingénieurs de mondes ", Les Cahiers du numérique 2/2003 (Vol. 4), p. 37-45. Consulté le 14/11/2013, URL : www.cairn.info/revue-les-cahiers-du-numerique-2003-2page-37.htm

12. Marc Marti, Art. cit., p. 82.

13. Bertrand Gervais, "Lecture de récits et compréhension de l'action ", Vox poetica, 2005, consulté le 21 novembre 2013, URL : http://www.vox-poetica.org/t/pas/bgervais.html

14. Baroni, Raphaël, «Compétences des lecteurs et schèmes séquentiels ", Littérature, 2005, $n^{\circ} 137$, pp.111-126, page 124, consulté le 3 décembre 2013, URL : http://www.persee.fr/web/revues/ home/prescript/article/litt_0047-4800_2005_num_137_1_1887

15. Nintendo, tous droits réservés, Source de l'image http://www.gamekyo.com

16. Sur ce phénomène, voir le travail universitaire de Philippe Mora, «Derrière l'E-Sport: un conflit d'experts de jeux réseaux compétitifs", in Les jeux vidéo : pratiques, contenus et enjeux sociaux, Paris, L'Harmattan, 2005, pp. 25-100. 
La presse hebdomadaire ou quotidienne contient quelques exemples du phénomène. Cécile Jandau « Jeu vidéo: un joueur de League of Legends obtient un visa sportif pour les Etats-Unis ", L'Express.fr, 16/08/2013, consulté le 21 novembre 2013, URL : http://www.lexpress.fr/culture/ jeu-video-un-joueur-de-league-of-legends-obtient-un-visa-sportif-pour-les-etats-

unis_1273821.html

17. Marc Marti, Art. cit., pp. 82-83.

18. Guillaume Fraissard, «Le cinéma mène les jeux », Le Monde.fr, 5/10/2013, consulté le 24 novembre 2013, URL: http://www.lemonde.fr/technologies/article/2013/10/05/le-cinemamene-les-jeux_3489118_651865.html

19. Site de lancement du jeu Wolfenstein New Order, consulté le 14 novembre 2013, URL : http:// www.wolfenstein.com/fr-fr

20. Antoine Dauphragne, «Le sens de la fiction ludique : jeu, récit et effet de monde ", Strence $n^{\circ} 2$, 2011, p.13, mis en ligne le 21 juin 2011, consulté le 18 novembre 2013. URL: http:// strenae.revues.org/312

21. Antoine Dauphragne, Art. cit., p. 14.

22. Pour la définition du concept en France voir Mélanie Bourdaa, "Le transmedia, entre narration augmentée et logiques immersives ", INA global, La revue des industries créatives et des médias, 18 juin 2012, consulté le 16 octobre 2012, URL: http://www.inaglobal.fr/numerique/ article/le-transmedia-entre-narration-augmentee-et-logiques-immersives

23. Voir Jeuxvideos.com, 2013, « D'abord l'argent, ensuite le pitch», 14 février 2013, consulté le 29 novembre 2013, URL: http://www.jeuxvideo.com/news/2013/00063992-d-abord-l-argentensuite-le-pitch.htm

24. Raphaël Baroni, Op. cit., p. 278.

25. Baptiste Campion, «Vers l'actualisation d'un " récit fantôme »? ", Communication, Vol. 26/2, 2008, p. 9, mis en ligne le 01 octobre 2009, consulté le 25 novembre 2013. URL: http:// communication.revues.org/824

26. Baptiste Campion, Art. cit., p. 13.

27. Régis Jaulin, Art. cit., p. 22.

28. Cécile Pearce, "L'émergence de la co-éditique, prochaine révolution interactive? ", Les défis du cybermonde, Presses de l'Université de Laval, Laval, 2003, pp. 173-193.

29. Philippe Mora, Art. cit., p. 52.

30. Cécile Pearce, Art. cit., p. 187.

31. Sur ce phénomène, voir Cécile Masoni-Lacroix, "Le transmedia: terrain d'acculturation communicationnelle des publics : vers une approche narratologique communicationnelle», Le Storytelling, succès des histoires, histoire d'un succès, Paris, L'Harmattan, 2012, pp. 53-71.

32. Nicolas Auray, Fanny Georges, «Les productions audiovisuelles des joueurs de jeux vidéo ", Réseaux 5/2012 ( $\mathrm{n}^{\circ} 175$ ), p. 145-173. Consulté le 18 novembre 2013, URL : www.cairn.info/revuereseaux-2012-5-page-145.htm

\section{ABSTRACTS}

Video games have recently become the object of major academic research and particularly on behalf of specialists of narratology. The first question that arises concerns of course the nature of the relationships between these two 
fields. We shall therefore try and define video game narrativity as a the combination of a "set-up" or "programmed" plot and the concrete experience of that plot by the player, that is, an experience which is both playful and narrative. On the basis of this preliminary work, we shall establish the existence of video game prototypes which might allow the researcher to describe various degrees of narrativity according to the function and the episode of the games.

The distinction between narrative prototypes will enable us to question and nuance the relations between the player and the play from the vantage point of narrativity but also to suggest a typology where the links between endo-narrativity and narrativity will take pride of place. This will eventually lead us to probe the concepts of immersion and interaction, which are crucial concepts for video games as well as for narratology.

Le jeu vidéo fait l'objet d'un intérêt récent de la part de la recherche universitaire, qui y voit un objet nouveau, en particulier pour la narratologie. La première question qui se pose est celle du rapport qu'entretient la narratologie avec ce nouvel objet. Nous essaierons ainsi de définir la narrativité vidéoludique comme la combinaison entre une intrigue «programmée » et matérielle et l'expérience de cette intrigue par le joueur, une expérience à la fois ludique et narrative.

A partir de ce constat, nous postulerons l'existence de prototypes vidéoludiques, qui permettraient de décrire des degrés de narrativité variables en fonction des jeux ou des moments de jeu.

La distinction de prototypes narratifs nous permettra d'approfondir les rapports entre le jeu et le joueur du point de vue de la narrativité, mais aussi d'établir une typologie où l'articulation entre l'endo-narratif et le narratif occupe une place importante. Elle nous sera utile pour approfondir la notion d'immersion et d'interaction, qui est essentielle dans les jeux vidéo mais aussi en narratologie.

\section{INDEX}

Geographical index: France, monde

Mots-clés: narrativité, récit interactif, jeu vidéo, typologie

Chronological index: XXème siècle, XXIème siècle

\section{AUTHOR}

MARC MARTI

Université Nice Sophia Antipolis, LIRCES, EA3159 